\title{
Densidad y módulo dinámico longitudinal de tres maderas angiospermas impregnadas con boro
}

\author{
Density and longitudinal dynamic modulus of three angiosperm woods impregnated with boron
}

Javier Ramón Sotomayor Castellanos*, Luz Elena Alfonsina Ávila Calderón
1 Universidad Michoacana de San Nicolás de Hidalgo. Morelia, Michoacán, México.

\section{RESUMEN}

El objetivo de la investigación fue determinar las densidades y los módulos dinámicos con vibraciones longitudinales, antes y después de aplicar el tratamiento de impregnación con sales de boro a madera de Spathodea campanulata, Fraxinus americana y Albizia plurijuga. Para cada especie se trabajaron 40 probetas que se impregnaron con el método de baño caliente-frío y se determinaron las retenciones correspondientes a concentraciones de $1 \%, 2 \%$ y $3 \%$. Las densidades de las maderas no se modifican después del tratamiento. Las densidades antes del tratamiento fueron $351 \mathrm{~kg} \mathrm{~m}^{-3}$ para S. campanulata, $654 \mathrm{~kg} \mathrm{~m}^{-3}$ para F. americana y $830 \mathrm{~kg} \mathrm{~m}^{-3}$ para A. plurijuga. Los módulos dinámicos de F. americana y A. plurijuga no variaron por el tratamiento. Caso diferente es $S$. campanulata cuyos módulos dinámicos sí variaron. Los módulos dinámicos antes del tratamiento fueron $4765 \mathrm{MN} \mathrm{m}^{-2}$ para S. campanulata, $13303 \mathrm{MN} \mathrm{m}^{-2}$ para F. americana y $16769 \mathrm{MN} \mathrm{m}^{-2}$ para A. plurijuga. Con base en sus módulos dinámicos las maderas de F. americana y $A$. plurijuga pueden usarse en diseño estructural. En cambio, $S$. campanulata puede emplearse como componente en productos donde no se requiera alta resistencia mecánica.

Palabras clave: Albizia plurijuga, baño caliente-frío, Fraxinus americana, Spathodea campanulata.

\section{ABSTRACT}

The objective of the investigation was to determine the densities and dynamic modules with longitudinal vibrations, before and after applying the impregnation treatment based on boron salts to wood from Spathodea campanulata, Fraxinus americana and Albizia plurijuga. For each species, 40 specimens were impregnated with the hot-cold bath method and the corresponding retentions were determined at concentrations of $1 \%, 2 \%$ and $3 \%$. Wood densities do not change after treatment. The densities before treatment were $351 \mathrm{~kg} \mathrm{~m}^{-3}$ for S. campanulata, $654 \mathrm{~kg} \mathrm{~m}^{-3}$ for F. americana and $830 \mathrm{~kg} \mathrm{~m}^{-3}$ for A. plurijuga. The dynamic modules of $F$. americana and A. plurijuga did not vary by treatment. A different case is $S$. campanulata whose dynamic modules did vary. The dynamic modules before treatment were $4765 \mathrm{MN} \mathrm{m}^{-2}$ for $S$. campanulata, $13303 \mathrm{MN} \mathrm{m}^{-2}$ for F. americana and $16769 \mathrm{MN}$ $\mathrm{m}^{-2}$ for A. plurijuga. Based on its dynamic modules, the woods of $F$. americana and A. plurijuga can be used in structural design. Instead, S. campanulata can be used as a component in products where high mechanical resistance is not required.
Key words: Albizia plurijuga, Fraxinus Americana, hot-cold bath, Spathodea campanulata.

\section{INTRODUCCIÓN}

El módulo de elasticidad de la madera de especies forestales está documentado en literatura internacional (Forest Products Laboratory, 2010) y en la bibliografía mexicana (Silva et al., 2010; Sotomayor, 2015; Tamarit y López, 2007). Este parámetro se utiliza en cálculo ingenieril y se determina en piezas de madera con dimensiones de empleo y bajo protocolos de normatividad. Sus valores son útiles en la industria de la edificación y de productos de madera (Thelandersson y Larsen, 2003; American Institute of Timber Construction, 2012). Sin embargo, para caracterizar maderas y potenciar usos alternos a los tradicionales, los módulos de elasticidad se determinan en probetas de pequeñas dimensiones (American Society for Testing Materials, 2014; International Organization for Standardization, 2014a).

Otra forma de determinar experimentalmente el módulo de elasticidad es bajo condiciones dinámicas empleando métodos no destructivos (Pellerin y Ross, 2002; Bucur, 2006). Esta tecnología aporta parámetros según los tipos de pruebas: el ultrasonido (Gonçalves et al., 2014; Dackermann et al., 2016), las ondas de esfuerzo (Neto et al., 2016; Freitas et al., 2016), las vibraciones transversales (Spycher et al., 2008; Kouroussis et al., 2017) y las vibraciones longitudinales (Chauhan y Sethy, 2016; Sotelo et al., 2017).

La madera es un material que se deteriora (Mattos et al., 2014; Bari et al., 2015; Beck et al., 2018). Para atenuar este fenómeno se han desarrollado técnicas de impregnación, principalmente, para evitar el deterioro producido por agentes biológicos (Henriques et al., 2014; Temiz et al., 2008) y la desintegración causada por el fuego (Wang et al., 2017; Jin y Chung, 2018). Entre las sustancias de impregnación más aplicadas, las sales de boro son una alternativa amigable con el medio ambiente (Obanda et al., 2008; Ahn et al., 2010).

La literatura especializada acerca del efecto de las sales de boro sobre la densidad y el módulo dinámico muestra resultados particulares a cada especie y según el método de impregnación aplicado. Baysal et al. (2007) y Keskin y Mutlu (2017) reportan que la densidad y el módulo dinámico aumentan como consecuencia del tratamiento. En cambio, Kartal et al. (2008) y Percin et al. (2015) informan que estos parámetros disminuyen. Este efecto se desconoce en la madera de Spathodea campanulata, Fraxinus americana y Albizia
*Autor para correspondencia: Javier Ramón Sotomayor Castellanos Correo electrónico: madera999@yahoo.com

Recibido: 28 de febrero de 2020

Aceptado: 17 de abril de 2020 
plurijuga. Con la perspectiva de utilizar mejor estos recursos maderables, generar información técnica para proteger la madera de estas especies y ampliar el conocimiento de sus características tecnológicas para el diseño de productos y estructuras de madera, se planteó el objetivo de determinar el efecto del tratamiento de preservación con sales de boro en la densidad y en el módulo dinámico de la madera de $S$. campanulata, F. americana y A. plurijuga.

\section{MATERIALES Y MÉTODOS}

Se trabajaron piezas de madera aserrada de S. campanulata, F. americana y A. plurijuga adquirida en establecimientos comerciales en el estado de Michoacán, México. La madera se acondicionó durante 12 meses en una cámara con temperatura de $20^{\circ} \mathrm{C}$ y humedad relativa de $65 \%$ hasta que alcanzó su contenido de humedad en equilibrio. Para cada especie se elaboraron 40 probetas con dimensiones de 0,02 $m \times 0,02 m$ de sección y 0,4 $m$ de largo, orientadas respectivamente en las direcciones radial, tangencial y longitudinal de la madera de acuerdo a la norma ISO 3129:2012 (International Organization for Standardization, 2012). Las probetas contenían solo madera de duramen y estaban libres de defectos.

Antes y después del tratamiento de impregnación se determinaron para cada especie el contenido de humedad y la densidad aparente en 40 probetas obtenidas de los mismos lotes de madera para las pruebas de vibraciones longitudinales con dimensiones de 0,02 $\mathrm{m} \times 0,02 \mathrm{~m}$ de sección y $0,06 \mathrm{~m}$ de largo. El contenido de humedad se determinó por el método de diferencia de pesos de acuerdo a la norma ISO 13061-1:2014 (International Organization for Standardization, 2014b). La densidad aparente de la madera se determinó de acuerdo a la norma ISO 13061-2:2014 (International Organization for Standardization, 2014c).

\section{Proceso de tratamiento de impregnación}

Las probetas se impregnaron utilizando el método denominado baño caliente-frío siguiendo el protocolo propuesto por Ávila et al. (2012). Se prepararon 30 litros de solución de trihidróxido de boro (ácido bórico, 39,4\%) y tetraborato de sodio (borato de sodio, $60,6 \%$ ) con tres concentraciones (C) $1 \%, 2 \%$ y $3 \%$, de acuerdo con la norma NMX-C-178-ONNCCE-2014 (Organismo Nacional de Normalización y Certificación de la Construcción y Edificación, 2014). Las probetas se sumergieron durante 8 horas en un baño de agua con temperatura de $60^{\circ} \mathrm{C}$, luego se sumergieron durante 16 horas en un baño frío con la solución a una temperatura de $23^{\circ} \mathrm{C}$.

Después de la impregnación en cada probeta se determinó la retención con la ecuación (1) (Simsek et al., 2013):

$$
\mathrm{R}=\frac{\left(\mathrm{P}_{\mathrm{DT}}-\mathrm{P}_{\mathrm{AT}}\right) \times \mathrm{C}}{\mathrm{V}_{\mathrm{DT}}}
$$

Donde:

$\mathrm{R}=$ Retención $\left(\mathrm{kg} \mathrm{m}^{-3}\right)$

$P_{D T}=$ Peso de la probeta después del tratamiento $(\mathrm{kg})$

$P_{A T}=$ Peso de la probeta antes del tratamiento $(\mathrm{kg})$

$C=$ Concentración de sales de boro

$\mathrm{V}_{\mathrm{DT}}=$ Volumen de la probeta después del tratamiento $\left(\mathrm{m}^{3}\right)$

\section{Pruebas de vibraciones longitudinales}

Las pruebas de vibraciones longitudinales consistieron en proporcionar un impacto en un extremo de la probeta, orientado hacia su dirección longitudinal (Figura 1) y siguiendo el procedimiento reportado por Yoshihara (2012). La probeta estuvo apoyada sobre un soporte elástico en medio de su longitud, correspondiente al nodo de su primer modo de vibración. Se midió la frecuencia natural del sistema en vibración, empleando el aparato Grindosonic ${ }^{\oplus}$ que capturó la señal de vibración por medio de un micrófono, posicionado en el extremo opuesto al del impacto. Se realizaron tres repeticiones y su promedio se tomó como el valor característico de una prueba. El módulo dinámico se calculó con la ecuación (2).

$$
\mathrm{E}_{\mathrm{L}}=4 \mathrm{~L}^{2} \rho_{\mathrm{CH}} \mathrm{f}^{2}
$$

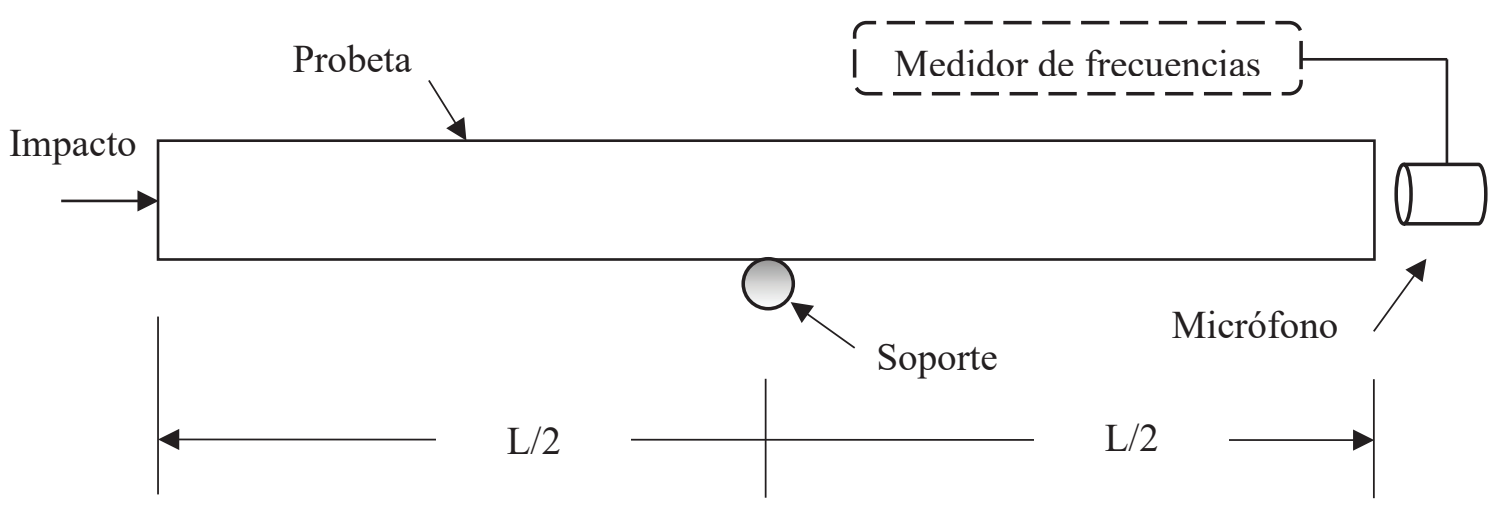

Figura 1. Esquema de las pruebas de vibraciones longitudinales. $L=$ Longitud de la probeta $(0,4 \mathrm{~m})$.

Figure 1. Scheme of longitudinal vibration tests. $L=$ Length of the specimen $(0,4 \mathrm{~m})$. 
Donde:

$\mathrm{E}_{\mathrm{L}}=$ Módulo dinámico $\left(\mathrm{N} \mathrm{m}^{-2}\right)$

$\mathrm{L}=$ Longitud de la probeta $(\mathrm{m})$

$\rho_{\mathrm{CH}}=$ Densidad $\left(\mathrm{kg} \mathrm{m}^{-3}\right)$

$\mathrm{f}=$ Frecuencia $(\mathrm{Hz})$

\section{Diseño experimental}

Se examinaron tres grupos de probetas de madera de las especies S. campanulata, F. americana y A. plurijuga. El factor de variación fue el tratamiento de impregnación con cuatro niveles: concentración de sales de boro de cero, uno, dos y tres por ciento. El nivel cero se consideró el grupo de control (testigo). Para cada especie y para cada concentración se formaron muestras independientes de diez probetas cada una, lo que dio un total de 120 probetas $(3 \times 4 \times 10)$.

Las variables de respuesta para dos estados antes del tratamiento AT y después del tratamiento DT fueron la retención de sales de boro $(R)$, la densidad $\left(\rho_{C H}\right)$ y el módulo dinámico $\left(\mathrm{E}_{\mathrm{oe}}\right)$. El contenido de humedad $(\mathrm{CH})$ solo se consideró como variable de referencia. Para cada variable se calculó la media $(\bar{x})$, desviación estándar $(\sigma)$ y el coeficiente de variación porcentual $(C V=\sigma / \bar{x})$. Se calcularon las correlaciones lineales y sus coeficientes de determinación $\left(R^{2}\right)$ antes y después del tratamiento entre los módulos dinámicos y las densidades.

\section{RESULTADOS Y DISCUSIÓN}

El contenido de humedad de la madera de S. campanulata fue del $8 \%(C V=14 \%)$, el de F. americana del $10 \%$ (CV $=6 \%$ ) y el de $A$. plurijuga del $12 \%(C V=5 \%)$. Con estos valores se consideró a la madera en estado seco y se asumió que el contenido de humedad no afectó las variables evaluadas.

\section{Retención}

Las magnitudes de las retenciones de sales de boro en las tres especies analizadas se incrementaron a medida que aumentó la concentración (Tabla 1). En cualquier nivel de concentración las retenciones fueron siempre mayores en E. campanulata, seguida de F. americana y A. plurijuga. Estos resultados pueden atribuirse al efecto de la densidad la madera de cada especie, en donde a menor valor en este parámetro el espacio vacío es mayor y por tanto es posible que la madera pueda retener un volumen mayor de solución impregnante (Tamarit y Fuentes, 2003). Las retenciones son comparables con los reportados por Percin et al. (2015) para Quercus partraea $\left(\rho_{\mathrm{CH}}=732 \mathrm{~kg} \mathrm{~m}^{-3} ; \mathrm{C}=5 \%\right.$ ) con retenciones de sales de boro entre $6,73 \mathrm{~kg} \mathrm{~m}^{-3}$ y 8,27 $\mathrm{kg} \mathrm{m}^{-3}$. Los valores promedio de retención para las especies, presentados en la Tabla 1, fueron mayores que la retención mínima de sales de boro sugerida por la Asociación Americana de Preservadores de Madera que es de 2,72 $\mathrm{kg} \mathrm{m}^{-3}$ (American Wood-Preservers' Association, 2007), misma que puede alcanzarse utilizando el nivel de concentración 2.

\section{Densidad}

La madera de $S$. campanulata exhibió la menor densidad promedio $\left(\rho_{\mathrm{CH}}=351 \mathrm{~kg} \mathrm{~m}^{-3}\right)$, F. americana la intermedia
Tabla 1. Valores de retenciones por nivel de concentración, densidades y módulos dinámicos para las especies evaluadas.

Table 1. Retention values by concentration level, densities and dynamic modules for the evaluated species.

\begin{tabular}{|c|c|c|c|c|c|c|}
\hline \multirow{3}{*}{ Especie } & \multirow[b]{2}{*}{ C } & \multirow[b]{2}{*}{$\mathbf{R}$} & \multicolumn{2}{|c|}{$\begin{array}{c}\text { Antes del } \\
\text { tratamiento }\end{array}$} & \multicolumn{2}{|c|}{$\begin{array}{l}\text { Después del } \\
\text { tratamiento }\end{array}$} \\
\hline & & & $\boldsymbol{\rho}_{\mathrm{CH}}$ & $E_{L}$ & $\boldsymbol{\rho}_{\mathrm{CH}}$ & $E_{L}$ \\
\hline & (\%) & $\left(\mathrm{kg} \mathrm{m}^{-3}\right)$ & $\left(\mathrm{kg} \mathrm{m}^{-3}\right)$ & $\left(\mathrm{MN} \mathrm{m}^{-2}\right)$ & $\left(\mathrm{kg} \mathrm{m}^{-3}\right)$ & $\left(\mathrm{MN} \mathrm{m}^{-2}\right)$ \\
\hline \multirow[t]{8}{*}{ S. campanulata } & 0\# & 0 & 351 & 4765 & 351 & 3099 \\
\hline & & & $(6,3)$ & $(47,5)$ & $(6,4)$ & $(50,8)$ \\
\hline & 1 & 2,3 & 363 & 3320 & 355 & 4423 \\
\hline & & & $(7,4)$ & $(41,1)$ & $(7,1)$ & $(12,0)$ \\
\hline & 2 & 4,2 & 343 & 2616 & 342 & 4703 \\
\hline & & & $(3,7)$ & $(79,1)$ & $(3,3)$ & $(31,8)$ \\
\hline & 3 & 6,4 & 347 & 1623 & 348 & 4277 \\
\hline & & & $(7,8)$ & $(50,2)$ & $(6,7)$ & $(13,2)$ \\
\hline \multirow[t]{8}{*}{ F. americana } & 0\# & 0 & 654 & 13303 & 658 & 13394 \\
\hline & & & $(5,6)$ & $(9,1)$ & $(5,7)$ & $(12,0)$ \\
\hline & 1 & 1,6 & 650 & 12268 & 647 & 13314 \\
\hline & & & $(4,4)$ & $(25,2)$ & $(4,4)$ & $(12,4)$ \\
\hline & 2 & 3,3 & 659 & 13626 & 656 & 13844 \\
\hline & & & $(7,3)$ & $(9,2)$ & $(7,6)$ & $(14,8)$ \\
\hline & 3 & 5,3 & 643 & 13612 & 645 & 14186 \\
\hline & & & $(5,3)$ & $(16,8)$ & $(5,1)$ & $(9,5)$ \\
\hline \multirow[t]{8}{*}{ A. plurijuga } & 0\# & 0 & 830 & 16769 & 830 & 16993 \\
\hline & & & $(2,7)$ & $(21,4)$ & $(2,7)$ & $(16,5)$ \\
\hline & 1 & 1,3 & 845 & 19046 & 841 & 18158 \\
\hline & & & $(3,2)$ & $(15,1)$ & $(3,3)$ & $(16,0)$ \\
\hline & 2 & 2,7 & 849 & 18319 & 846 & 18280 \\
\hline & & & $(3,2)$ & $(21,8)$ & $(3,3)$ & $(19,7)$ \\
\hline & 3 & 3,7 & 827 & 17382 & 827 & 17153 \\
\hline & & & $(4,6)$ & $(15,3)$ & $(4,7)$ & $(14,2)$ \\
\hline
\end{tabular}

$\mathrm{C}=$ Concentración; $\mathrm{R}=$ Retención; $\rho_{\mathrm{CH}}=$ Densidad; $\mathrm{E}_{\mathrm{L}}=$ Módulo dinámico; Coeficiente de variación entre paréntesis; \# = Testigo.

$\left(\rho_{C H}=652 \mathrm{~kg} \mathrm{~m}^{-3}\right)$ y A. plurijuga la más alta $\left(\rho_{\mathrm{CH}}=838 \mathrm{~kg} \mathrm{~m}^{-3}\right)$ (Tabla 1). Las densidades no presentan variaciones importantes respecto a las muestras de control sin tratamiento $(C=$ $0 \%)$ ni entre las retenciones resultantes de las tres concen- 


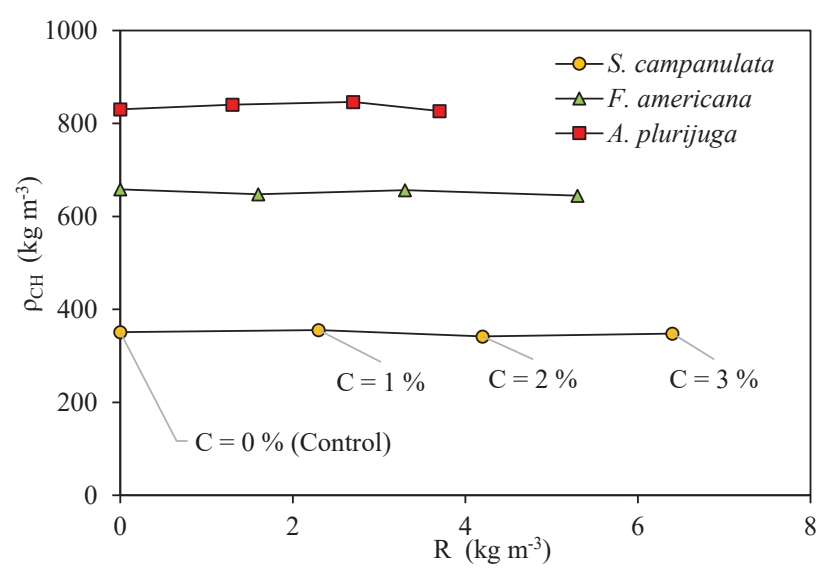

Figura 2. Comportamiento de la densidad $\left(\rho_{\mathrm{CH}}\right)$ en función de la retención (R) por especie.

Figure 2. Density $\left(\rho_{C H}\right)$ behavior as a function of retention (R) by species.

traciones (Figura 2). La evidencia indica que las densidades de estas maderas no se modifican después del tratamiento con sales de boro, empleando el método de baño calientefrío. Percin et al. (2015) reporta variaciones en la densidad de la madera ocasionadas principalmente por el peso de las sales de boro, la diversidad en la porosidad de la especie en estudio $y$, cuando es el caso, el efecto de la temperatura empleada para impregnar la madera.

\section{Módulo dinámico}

El menor valor del módulo dinámico lo presentó $S$. campanulata (3081 $\mathrm{MN} \mathrm{m}^{-2}$ ) y el máximo A. plurijuga (17879

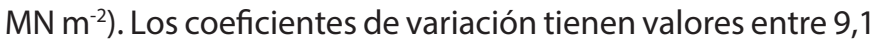
$\%$ para F. americana y $21,8 \%$ para A. plurijuga (Tabla 1 ). Caso excepcional fue $S$. campanulata con coeficientes de variación entre 41,1 y $79,1 \%$.

Los módulos dinámicos presentan diferencias importantes entre las muestras de control sin tratamiento $(C=0 \%)$ y las muestras tratadas con boro (Figura 3). Excluyendo los resultados de $S$. campanulata, las magnitudes de los módulos

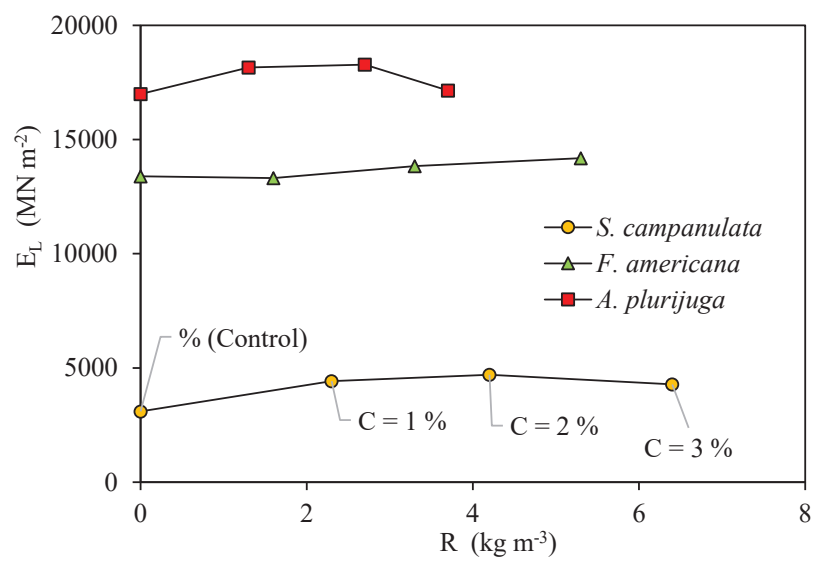

Figura 3. Comportamiento del módulo dinámico $\left(E_{\mathrm{L}}\right)$, en función de la retención (R) por especie.

Figure 3. Behavior of the dynamic module $\left(E_{L}\right)$, as a function of retention (R) by species. de elasticidad y sus coeficientes de variación son similares a los reportados por Baar et al. (2015). Los autores reportan módulos dinámicos determinados por vibraciones longitudinales para Afzelia bipindensis de $12860 \mathrm{MN} \mathrm{m}^{-2}\left(\mathrm{CV}=18 \% ; \rho_{\mathrm{CH}}\right.$ $\left.=766 \mathrm{~kg} \mathrm{~m}^{-3}\right)$; Intsia bijuga de $18220 \mathrm{MN} \mathrm{m}^{-2}\left(\mathrm{CV}=13 \%\right.$; $\rho_{\mathrm{CH}}$ $\left.=813 \mathrm{~kg} \mathrm{~m}^{-3}\right)$; Millettia laurentii de $16570 \mathrm{MN} \mathrm{m}^{-2}(\mathrm{CV}=15 \%$; $\left.\rho_{\mathrm{CH}}=856 \mathrm{~kg} \mathrm{~m}^{-3}\right)$; Astronium graveolens de $17920 \mathrm{MN} \mathrm{m}^{-2}$ (CV $\left.=12 \% ; \rho_{C H}=840 \mathrm{~kg} \mathrm{~m}^{-3}\right)$; y para Microberlinia brazzavillensis de $19620 \mathrm{MN} \mathrm{m}^{-2}\left(\mathrm{CV}=15 \%\right.$; $\left.\rho_{\mathrm{CH}}=810 \mathrm{~kg} \mathrm{~m}^{-3}\right)$.

Las magnitudes de los módulos dinámicos clasifican las maderas de $F$. americana y A. plurijuga (Tabla 1 ) en el grupo I de valores especificados de módulos de elasticidad correspondiente al $5^{\circ}$ percentil de maderas de especies latifoliadas en el Reglamento de Construcciones para el Distrito Federal y sus Normas Técnicas Complementarias para Diseño y Construcción de Estructuras de Madera (Gobierno del Distrito Federal, 2004). No así para S. campanulata cuyos módulos dinámicos son menores que los requeridos por este reglamento. Sin embargo, los valores de la Tabla 1 son derivados de pruebas dinámicas en probetas de pequeñas dimensiones y libres de defectos. De tal forma que para emplear estas maderas en cálculo estructural es necesario determinar experimentalmente sus módulos de elasticidad en piezas de tamaño estructural y ajustarlos con los factores que recomiendan el reglamento citado.

La densidad determinada en las especies evaluadas es un índice confiable para estimar su resistencia mecánica (Baar et al., 2015), este hecho se explica por la alta correlación entre el módulo dinámico y la densidad de la madera, antes del tratamiento de impregnación, dado su excelente valor en el coeficiente de determinación (Figura 4), condición que se ratifica por la correlación referida entre ambas variables después del proceso de impregnación (Figura 5). La impregnación conserva la magnitud de las densidades de F. americana y A. plurijuga, y no modifica sus módulos de elasticidad. En cambio, los resultados para S. campanulata señalan que el tratamiento modificó sus módulos de elasticidad de una manera importante.

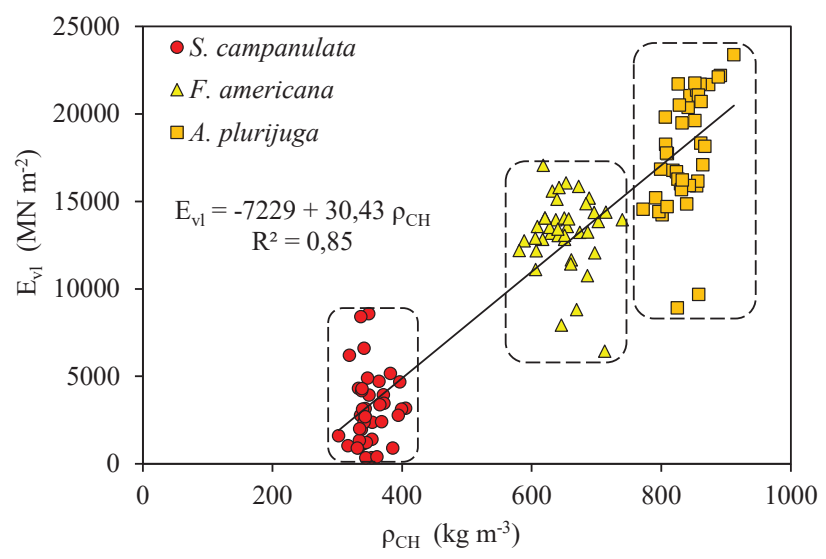

Figura 4. Correlación del módulo dinámico $\left(\mathrm{E}_{\mathrm{L}}\right)$ en función de la densidad $\left(\rho_{\mathrm{CH}}\right)$ antes del tratamiento de impregnación con sales de boro.

Figure 4. Correlation of the dynamic modulus $\left(E_{\mathrm{L}}\right)$ as a function of the density $\left(\rho_{C H}\right)$ before the impregnation treatment with boron salts. 


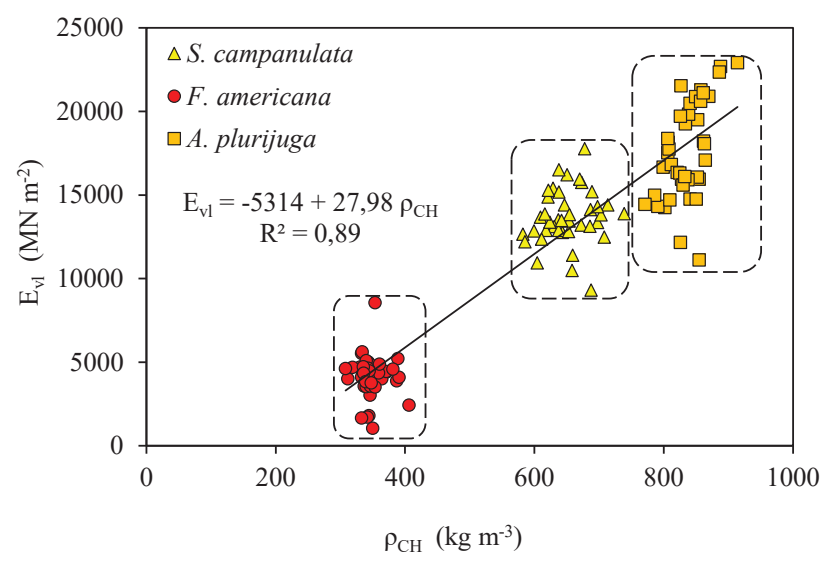

Figura 5. Correlación del módulo dinámico $\left(\mathrm{E}_{\mathrm{L}}\right)$ en función de la densidad $\left(\rho_{\mathrm{CH}}\right)$ después del tratamiento de impregnación con sales de boro.

Figure 5. Correlation of dynamic modulus $\left(E_{L}\right)$ as a function of density $\left(\rho_{C H}\right)$ after impregnation treatment with boron salts.

\section{CONCLUSIONES}

El método de baño caliente-frío es eficiente para impregnar con sales de boro las maderas de S. campanulata, $F$. americana y A. plurijuga. Las retenciones se incrementaron a medida que las concentraciones aumentan. En cambio, disminuyen a medida que la densidad de las maderas aumenta.

Las pruebas de vibraciones longitudinales permiten determinar los efectos del impregnado en los módulos de elasticidad. El método de impregnación no modificó las densidades y módulos dinámicos en F. americana y A. plurijuga, en tanto que los módulos dinámicos variaron para S. campanulata. Se confirmó que los módulos dinámicos de las tres maderas aumentan en función de su densidad.

Con base en sus módulos dinámicos las maderas de $F$. americana y A. plurijuga pueden usarse en diseño estructural. En cambio, S. campanulata puede emplearse como componente en productos donde no se requiera alta resistencia mecánica.

\section{REFERENCIAS}

Ahn, S.H., Oh, S.C., Choi, I.G., Han, G.S., Jeong, H.S., Kim, K.W., Yoon, Y.H. y Yang I. 2010. Environmentally friendly wood preservatives formulated with enzymatic-hydrolyzed okara, copper and/or boron salts. Journal of Hazardous Materials. 178(1): 604-611.

American Institute of Timber Construction. 2012. Timber Construction Manual. American Institute of Timber Construction, Tigar.

American Society for Testing Materials. 2014. ASTM D143-14. Standard Test Methods for Small Clear Specimens of Timber. American Society for Testing Materials, West Conshohocken.

American Wood-Preservers' Association. 2007. P5-07 Standard for Waterborne Preservatives. American Wood-Preservers' Association, Chicago.

Ávila Calderón, L.E.A., Herrera Ferreyra, M.A. y Raya González, D. 2012. Preservación de la Madera en México. Universidad Michoacana de San Nicolás de Hidalgo, Morelia.

Baar, J., Tippner, J. y Rademacher, P. 2015. Prediction of mechanical properties modulus of rupture and modulus of elasticity of five tropical species by nondestructive methods. Maderas. Ciencia y tecnología. 17(2): 239-252.

Bari, E., Nazarnezhad, N., Kazemi, S.M., Tajick G., Mohammad A., Mohebby, B., Schmidt, O. y Clausen, C.A. 2015. Comparison between degradation capabilities of the white rot fungi Pleurotus ostreatus and Trametes versicolor in beech wood. International Biodeterioration \& Biodegradation. 104: 231 237.

Baysal, E., Yalinkilic, M.K., Altinok, M., Sonmez, A., Peker, H. y Colak, M. 2007. Some physical, biological, mechanical, and fire properties of wood polymer composite (WPC) pretreated with boric acid and borax mixture. Construction and Building Materials. 21(9): 1879-1885.

Beck, G., Thybringc, E.E. y Thygesenc, L.G. 2018. Brown-rot fungal degradation and de-acetylation of acetylated wood. International Biodeterioration \& Biodegradation. 135: 62-70.

Bucur, V. 2006. Acoustics of Wood. Springer-Verlag, Berlin.

Chauhan, S. y Sethy, A. 2016. Differences in dynamic modulus of elasticity determined by three vibration methods and their relationship with static modulus of elasticity. Maderas: Ciencia y Tecnología. 18(2): 373-382.

Dackermann, U., Elsener, R., Li, J. y Crews, K. 2016. A comparative study of using static and ultrasonic material testing methods to determine the anisotropic material properties of wood. Construction and Building Materials. 102: 963-976.

Forest Products Laboratory. 2010. Wood handbook. Wood as an engineering material. Forest Products Laboratory, Madison.

Freitas, A.S., Gonçalez, J.C. y Del Menezzi, C.H. 2016. Tratamento termomecânico e seus efeitos nas propriedades da Simarouba amara (Aubl.). Floresta e Ambiente. 23(4): 565572.

Gobierno del Distrito Federal. 2004. Reglamento de Construcciones para el Distrito Federal y sus Normas Técnicas Complementarias para Diseño y Construcción de Estructuras de Madera. Gobierno del Distrito Federal, México.

Gonçalves, R., Trinca, A.J. y Pellis, B.P. 2014. Elastic constants of wood determined by ultrasound using three geometries of specimens. Wood Science and Technology. 48(2): 269-287.

Henriques, D., De Brito, J., Duarte, S. y Nunes, L. 2014. Consolidating preservative-treated wood: Combined mechanical performance of boron and polymeric products in wood degraded by Coniophora puteana. Journal of Cultural Heritage. 15(1): 10-17.

International Organization for Standardization. 2012. ISO 3129:2012. Wood. Sampling methods and general requirements for physical and mechanical testing of small clear wood specimens. International Organization for Standardization, Geneva.

International Organization for Standardization. 2014a. ISO 13061-4:2014. Physical and mechanical properties of wood. Test methods for small clear wood specimens. Part 4: determination of modulus of elasticity in static bending. International Organization for Standardization, Geneva.

International Organization for Standardization. 2014b. ISO 13061-1:2014. Physical and mechanical properties of wood. Test methods for small clear wood specimens. Part 1: determination of moisture content for physical and mechanical tests. International Organization for Standardization, Geneva. 
International Organization for Standardization. 2014c. ISO 13061-2:2014. Physical and mechanical properties of wood. Test methods for small clear wood specimens. Part 2: determination of density for physical and mechanical tests. International Organization for Standardization, Geneva.

Jin, E. y Chung, Y.J. 2018. Fire risk of wood treated with boron compounds by combustion test. Fire Science and Engineering. 32(3): 19-26.

Kartal, S.N., Hwang, W.J. y Imamura, Y. 2008. Combined effect of boron compounds and heat treatments on wood properties: Chemical and strength properties of wood. Journal of Materials Processing Technology. 198(1): 234-240.

Keskin, H. y Mutlu, E. 2017. Impacts of impregnation with fire retardant chemicals on the moe in bending of some woods. Journal of Polytechnic. 20(3): 607-612.

Kouroussis, G., Ben, F.L. y Descamps, T. 2017. Assessment of timber element mechanical properties using experimental modal analysis. Construction and Building Materials. 134: 254-261.

Mattos, B.D., De Cademartori, P.H.G., Lourençon, T.V., Gatto, D.A. y Magalhães, W.L.E. 2014. Biodeterioration of wood from two fast-growing eucalypts exposed to field test. International Biodeterioration \& Biodegradation. 93: 210-215.

Neto, P.N.M., Paes, J.B. y Segundinho, P.G.D. 2016. Evaluation of elasticity and rupture modulus of woods by destructive and non-destructive techniques. Scientia Forestalis. 44(111): 683-690.

Obanda, D.N., Shupe, T.F. y Barnes, H.M. 2008. Reducing leaching of boron-based wood preservatives. A review of research. Bioresource Technology. 99(15): 7312-7222.

Organismo Nacional de Normalización y Certificación de la Construcción y Edificación. 2014. NMX-C-178-ONNCCE-2014: Industria de la construcción. Preservadores para madera. Clasificación y requisitos. México.

Pellerin, R.F. y Ross, R.J. 2002. Nondestructive evaluation of wood. Forest Products Society, Madison.

Percin, O., Sofuoglu, S.D. y Uzun, O. 2015. Effects of boron impregnation and heat treatment on some mechanical properties of oak (Quercus petraea Liebl.) wood. BioResources. 10(3): 3963-3978.
Silva Guzmán, J.A., Fuentes Talavera, F.J., Rodríguez Anda, R., Torres Andrade, P.A., Lomelí Ramírez, M.A., Ramos Quirarte, J., Waitkus, C. y Richter, H.G. 2010. Fichas de propiedades tecnológicas y usos de maderas nativas de México e importadas. Comisión Nacional Forestal, México.

Simsek, H., Baysal, E., Yilmaz, M.y Culha, F. 2013. Some mechanical properties of wood impregnated with environmentallyfriendly boron and copper based chemicals. Wood Research. 58(3): 495-504.

Sotelo Montes., C., Weber, J.C., Garcia, R.A., Silva, D.A. y Muñiz, G.I.B. 2017. Variation in growth, wood stiffness and density, and correlations between growth and wood stiffness and density in five tree and shrub species in the Sahelian and Sudanian ecozones of Mali. Trees. 31(3): 833-849.

Sotomayor Castellanos, J.R. 2015. Banco FITECMA de características físico-mecánicas de maderas mexicanas. Universidad Michoacana de San Nicolás de Hidalgo, Morelia.

Spycher, M., Schwarze, F.W.M.R. y Steiger, R. 2008. Assessment of resonance wood quality by comparing its physical and histological properties. Wood Science and Technology. 42(4): 325-342.

Tamarit Urias, J.C. y López Torres, J.L. 2007. Xilotecnología de los principales árboles tropicales de México. Instituto Nacional de Investigaciones Forestales, Agrícolas y Pecuarias, México.

Tamarit Urias, J.C. y Fuentes Salinas M. 2003. Parámetros de humedad de 63 maderas latifoliadas mexicanas en función de su densidad básica. Revista Chapingo Serie Ciencias Forestales y del Ambiente. 9(2): 155-164.

Temiz, A., Alfredsen, G., Eikenes, M. y Terziev, N. 2008. Decay resistance of wood treated with boric acid and tall oil derivates. Bioresource Technology. 99(7): 2102-2106.

Thelandersson, S. y Larsen H.J. 2003. Timber Engineering. Wiley, New Jersey.

Wang, F., Liu, J. y Lv, W. 2017. Thermal degradation and fire performance of wood treated with PMUF resin and boron compounds. Fire and Materials. 41(8): 1051-1057.

Yoshihara, H. 2012. Examination of the specimen configuration and analysis method in the flexural and longitudinal vibration tests of solid wood and wood-based materials. Forest Products Journal. 62(3): 191-200. 